\title{
Groove Pancreatitis
}

\author{
Koji Tezuka Takatoshi Makino Ichiro Hirai Wataru Kimura
}

First Department of Surgery, Yamagata University School of Medicine, Yamagata, Japan

\section{Key Words}

Alcohol abuse - Common bile duct - Groove pancreatitis . Intraduodenal cyst • Pancreatoduodenectomy

\begin{abstract}
Groove pancreatitis is a segmental chronic pancreatitis that affects the anatomical area between the pancreatic head, the duodenum, and the common bile duct, referred to as the groove area. Most patients with groove pancreatitis are males aged 40-50 years with a history of alcohol abuse. In about $20 \%$ of patients undergoing pancreaticoduodenectomy to treat chronic pancreatitis, groove pancreatitis is detected. The clinical symptoms are weight loss, upper abdominal pain, postprandial vomiting, and nausea due to duodenal stenosis. The pathogenesis of groove pancreatitis is thought to be anatomical or functional obstruction of the minor papilla. The viscosity of pancreatic juice increases due to excessive alcohol consumption and/or smoking, leading to calcification of the pancreatic duct. According to these conditions, pancreatitis in the groove area might arise due to impaired pancreatic juice outflow. The descending part of the duodenum is usually stenotic. Severe fibrosis and scarring are evident in the groove area. Characteristic pathological findings are cystic lesions in the duodenal wall, Brunner gland hyperplasia, dilation of Santorini's duct and protein plaques in the pancreatic duct. A differential diagnosis of groove pancreatitis from peripancreatic cancer is clinically important. Cystic lesions in the duodenal wall and smooth stenosis of the bile duct are important findings of groove pancreatitis revealed by endoscopic ultrasonography, computed tomography and magnetic resonance imaging. Biop-
\end{abstract}

sy through the duodenum is also useful for diagnosis. Conservative treatment options include endoscopic stenting of the minor papilla, but long-term outcomes remain unclear. Pancreatoduodenectomy is a rational treatment for symptomatic groove pancreatitis. Copyright $\odot 2010$ S. Karger AG, Basel

\section{Introduction}

Groove pancreatitis is a segmental chronic pancreatitis that affects the anatomical area between the pancreatic head, the duodenum, and the common bile duct, which is referred to as the groove area. In 1973, Becker [1] first used the German term 'Rinnenpankreatitis' to describe segmental pancreatitis of the groove area and this was later translated into groove pancreatitis by Stolte et al. [2] in 1982.

Adsay and Zamboni [3] recently proposed the name paraduodenal pancreatitis to find a common term for groove pancreatitis, cystic dystrophy of the heterotopic pancreas [4], periampullary duodenal wall cyst [5], pancreatic hamartoma of the duodenal wall [6], and myoadenomatosis [7], because the pathological features of these diseases overlap.

Stolte et al. [2] classified groove pancreatitis into a pure form, in which scarring is localized to the groove area, and a segmental form, in which scarring extends to the dorsocranial portion of the pancreatic head. These forms accounted for 8.9 and $15.5 \%$, respectively, of 123 pancreaticoduodenectomies performed on patients with chronic pancreatitis [2].

\section{KARGER}

Fax +4161306 1234

E-Mail karger@karger.ch

www.karger.com
(C) 2010 S. Karger AG, Basel

0253-4886/10/0272-0149\$26.00/0

Accessible online at:

www.karger.com/dsu
Wataru Kimura, MD, PhD

First Department of Surgery, Yamagata University School of Medicine

2-2-2 Iida-Nishi

Yamagata 990-9585 (Japan)

Tel. +81 23 628 5336, Fax +81 23628 5339, E-Mail wkimura@ med.id.yamagata-u.ac.jp 
Because of its rarity, the distinct incidence of groove pancreatitis is unknown, but it accounts for 19.5-24.4\% of pancreaticoduodenectomies performed to treat chronic pancreatitis $[2,8]$. Most patients with groove pancreatitis are male, aged $40-50$ years, and have a history of alcohol abuse $[2,8]$. A case report is reported in the Appendix.

\section{Pathogenesis}

The pathogenesis of groove pancreatitis remains unclear. Anatomical or functional obstruction of the minor papilla is one of the issues considered. The viscosity of the pancreatic juice changes following excessive alcohol consumption and/or smoking, leading to pancreatic duct calcification. These conditions suggest that pancreatitis in the groove area might arise due to outflow obstruction of the pancreatic juice. Brunner gland hyperplasia is considered to arise from an increase in cholecystokinin-pancreozymin or gastrin levels. Stasis of the pancreatic juice in the dorsal pancreas caused by Brunner gland hyperplasia leads to pancreatitis in the groove area [2].

The dorsal pancreas sometimes projects embryologically into the duodenal lumen. This anatomical variation leads to dysfunction of the minor papilla [9]. Anatomical factors, including a duodenal bud and ectopic pancreas, are other possible reasons for groove pancreatitis [10]. Moreover, a history of gastrectomy, gastroduodenal ulcer, and biliary disease might also be pathogenetic factors [2]

\section{Clinical Symptoms}

The clinical manifestations of groove pancreatitis comprise weight loss, upper abdominal pain, postprandial vomiting, and nausea due to duodenal stenosis that can range from a few weeks to several years $[3,11,12]$, but jaundice is rare.

\section{Pathology}

Thickening and scarring of the duodenal wall close to the minor papilla usually causes stenosis of the second portion of the duodenum. Cystic changes in the thickened duodenal wall are characteristic. Cysts from 0.2 to $2 \mathrm{~cm}$ develop in the submucosa and muscle layer, and contain clear fluid as well as occasional granular white material and stones $[9,10]$. Becker and Mischke [8] have identified intraduodenal cysts in $49 \%$ of patients with groove pancreatitis.

The cyst wall is covered with columnar epithelium or replaced by granulation tissue [10]. Nodular lesions, ulceration, and scarring are gross features of the duodenal mucosa. Microscopy reveals Brunner gland hyperplasia and thickening of the submucosal and muscle layers caused by extensive fibrosis [10].

Fibrosis and scarring are obvious in the groove area $[2$, 3]. The parenchyma of the pancreatic head is initially not involved, but with disease progression, fibrosis spreads to the parenchyma of the pancreatic head and causes dilation of the main pancreatic duct [10]. Santorini's duct can become dilated with protein plugs and calcification $[2,8$, 13].

Groove pancreatitis can be classified into a pure form, which is restricted to the groove area without involvement of the main pancreatic duct, and a segmental form with pancreatitis, which encompasses the entire head of the pancreas, with a stenotic and dilated main pancreatic duct. Fibrosis around the inferior bile duct causes scarring [2] or lymph node involvement around the pancreas [10].

\section{Diagnosis}

Serum pancreatic (amylase, lipase, and elastase 1) and hepatic enzyme levels are sometimes slightly elevated [11, 14]. Serum levels of carcinoembryonic antigen and carbohydrate antigen (CA 19-9) are usually normal [13].

Upper gastrointestinal fiberscopy shows erosion redness, stenosis due to edema, and a polypoid appearance in the descending part of the duodenum [15]. Transduodenal biopsy is important to distinguish peripancreatic cancer from groove pancreatitis $[12,15]$.

Ultrasonography shows the entire thickness of the duodenal wall and stenosis of the lumen, with a hypoechoic lesion between the pancreas head and the thickened duodenum [15]. Endoscopic ultrasonography reveals smooth stenosis of the common bile duct; Santorini's duct is usually undetectable. Duct-penetrating signs are evident in the irregular hypoechoic mass [12].

Dynamic CT shows a low-density area in the early phase due to a large-fiber fibrosis component. Enhancement is delayed during the late phase of dynamic CT [16]. Cystic lesions and a thickened duodenal wall are also useful indicators of groove pancreatitis on dynamic CT images [16]. 
MRI shows a sheet-like mass in the groove area, which is hypointense relative to the pancreatic parenchyma on $\mathrm{T}_{1}$-weighted images, and iso- to slightly hypointense on $\mathrm{T}_{2}$-weighted images [17]. Such masses that are hyperintense on $\mathrm{T}_{2}$-weighted images at the subacute inflammatory period become hypointense due to fibrosis, but the parenchyma of the pancreatic head is hyperintense. Grooves are revealed as hypointense areas on $\mathrm{T}_{1}$-weighted images [18]. Dynamic MRI usually shows delayed enhancement similar to dynamic CT [19]. $\mathrm{T}_{2}$-weighted imaging is useful to detect intraduodenal wall cysts [16].

Magnetic resonance cholangiopancreatography shows a widened space between the duodenum, common bile duct, and distal pancreas [20]. The common bile duct gradually tapers, being in contrast to the abrupt stenosis characterizing pancreatic cancer [20]. The diameter of the main pancreatic duct is normal or slightly tapered [21]. When the duodenum of patients with groove pancreatitis is too narrow to perform gastrointestinal fiberscopy, magnetic resonance cholangiopancreatography is a useful diagnostic option.

Endoscopic retrograde cholangiopancreatography also reveals tapering of the lower bile duct $[1,12,15]$. An irregularly strictured bile duct suggests rather cancer than groove pancreatitis. Dilation of the Santorini duct or its branches, pancreatic stones or protein plaques are sometimes detectable $[8,12,13]$.

\section{Differential Diagnosis}

Clinicians must consider peripancreatic cancer for a differential diagnosis of groove pancreatitis. Other differential diseases include autoimmune pancreatitis and duodenal hamartoma [10]. Groove pancreatitis should always be considered when a patient has a history of excessive alcohol consumption.

Findings that differ from those of pancreatic carcinoma are as follows: groove pancreatitis appears as a sheetlike mass, whereas pancreatic carcinoma manifests as a round irregular mass. Stenosis of the bile duct is smooth and long in groove pancreatitis, but abrupt and short in pancreatic cancer. Cystic lesions are more often present in the duodenal wall in groove pancreatitis than in pancreatic carcinoma. However, arteries in pancreatic head lesions are often encased in pancreatic carcinoma but rarely in groove pancreatitis [21].

Because some conditions closely resemble pancreatic carcinoma, careful follow-up of patients with a diagnosis of groove pancreatitis is necessary.

\section{Treatment}

In patients with groove pancreatitis, resection is often performed for the diagnosis of a peripancreatic malignancy [13]. However, understanding of groove pancreatitis has recently increased and patients with symptoms such as abdominal pain are surgically treated [22].

Conservative treatment options are to stop smoking/ alcohol consumption, recovery of pancreatic function, and analgesics. However, such treatments are only temporarily effective as a rule [20]. One report has described curing groove pancreatitis by endoscopic stenting of the minor papilla, but the long-term clinical course remains obscure [23].

Surgery is the treatment of choice when symptoms do not improve, or the condition is difficult to distinguish from pancreatic carcinoma. Surgical procedures comprise pancreatoduodenectomy and pylorus-preserving pancreatoduodenectomy. Casetti et al. [22] preferred pancreatoduodenectomy to pylorus-preserving pancreatoduodenectomy due to inflammation around the pylorus. In a study by Rahman et al. [18], body weight increased and chronic abdominal pain was relieved in all patients who underwent pancreatoduodenectomy. Complete pain relief was reported in $76 \%$ of the patients after pancreatoduodenectomy, with the remainder having occasionally relapsing abdominal pain [22].

\section{Appendix: Case Report}

A 55-year-old male smoker who consumed excessive amounts of alcohol presented with upper abdominal pain diagnosed as chronic pancreatitis. Laboratory data showed slightly elevated serum levels of amylase, lipase and elastase-1 (150 IU/l, $118 \mathrm{U} / 1$ and $348 \mathrm{ng} / \mathrm{dl}$, respectively). A CT scan showed a thickened duodenal wall and an enlarged pancreatic head (fig. 1). Duodenography revealed a $3-\mathrm{cm}$ stenosis of the descending part of the duodenum. Upper gastrointestinal fiberscopy revealed edema of the duodenal mucosa and stenosis of the duodenum, and endoscopic retrograde cholangiopancreatography showed a left shift of the common bile duct as well as a disrupted Santorini duct. Groove pancreatitis was diagnosed by pancreatoduodenectomy. The resected specimen revealed a cystic lesion in the duodenal wall, as well as severe fibrosis and inflammatory cell infiltration in the groove area (fig. 2). The patient is doing well without recurrence of pancreatitis. 


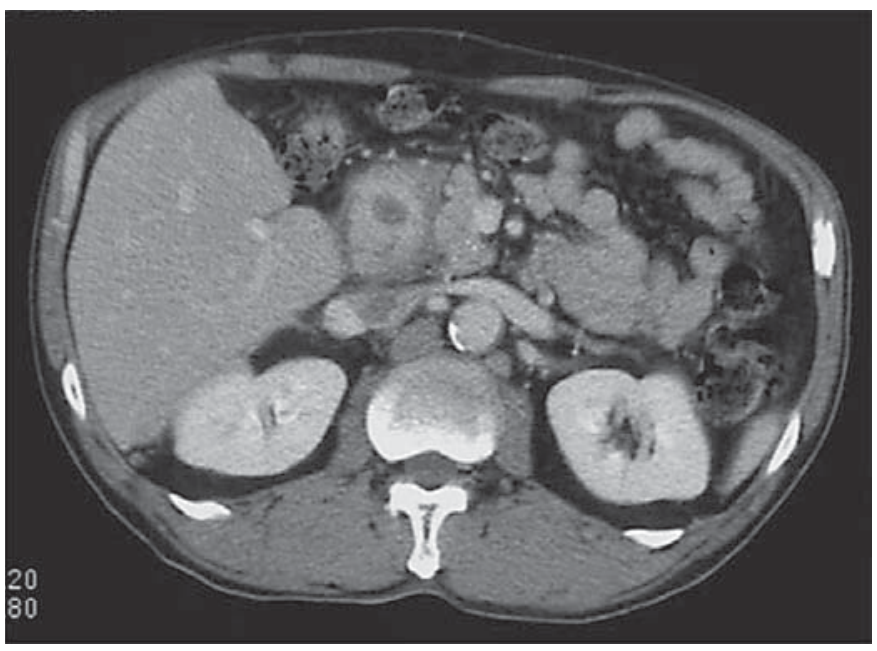

Fig. 1. CT scan of a patient with groove pancreatitis revealed a thickened duodenal wall and an enlarged pancreatic head.

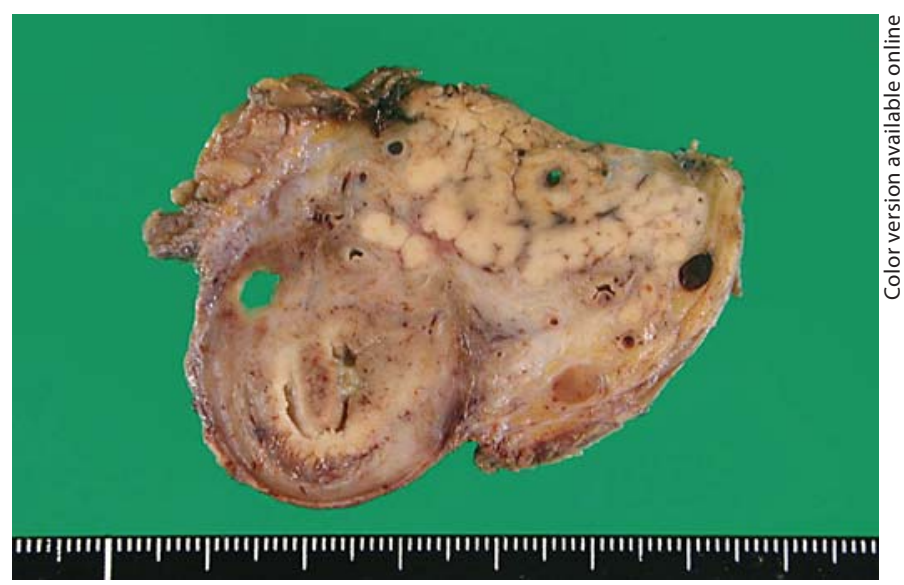

Fig. 2. Macroscopic appearance of the resected specimen showed a cystic lesion in the duodenal wall and evidence of severe fibrosis and inflammatory cell infiltration in the groove area.

\section{References}

1 Becker V: Bauchspeicheldrüse; in Doerr W, Seifert G, Uhlinger E (eds): Spezielle pathologische Anatomie. Berlin, Springer, 1973, vol 4.

2 Stolte M, Weiss W, Volkholz H, Rösch W: A special form of segmental pancreatitis: 'groove pancreatitis'. Hepatogastroenterology 1982;29:198-208.

3 Adsay NV, Zamboni G: Paraduodenal pancreatitis: a clinico-pathologically distinct entity unifying 'cystic dystrophy of heterotopic pancreas', 'para-duodenal cyst', and 'groove pancreatitis'. Semin Diagn Pathol 2004;21: 247-254.

4 Potet F, Duclert N: Cystic dystrophy on aberrant pancreas of the duodenal wall. Arch Fr Mal App Dig 1970;59:223-238.

5 Solcia E, Capella C, Klöppel G: Tumors of the Pancreas. AFIP Atlas of Tumor Pathology Third Series, Fascicle 20. Washington, Armed Forces Institute of Pathology, 1997.

-6 McFaul CD, Vitone LJ, Campbell F, Azadeh B, Hughes ML, Garvey CJ, Ghaneh P, Neoptolemos JP: Pancreatic hamartoma. Pancreatology 2004;4:533-538.

7 Bill K, Belber JP, Carson JW: Adenomyoma (pancreatic heterotopia) of the duodenum producing common bile duct obstruction. Gastrointest Endosc 1982;28:182-184.

8 Becker V, Mischke U: Groove pancreatitis. Int J Pancreatol 1991;10:173-182.
Ohta T, Nagakawa T, Kobayashi H, Kayahara M, Ueno K, Konishi I, Miyazaki I: Histomorphological study on the minor duodenal papilla. Gastroenterol Jpn 1991;26:356-362.

10 Zamboni G, Capelli P, Scarpa A, Bogina G, Pesci A, Brunello E, Klöppel G: Nonneoplastic mimickers of pancreatic neoplasms. Arch Pathol Lab Med 2009;133:439-453.

11 Shudo R, Obara T, Tanno S, Fujii T, Nishino N, Sagawa M, Ura H, Kohgo Y: Segmental groove pancreatitis accompanied by protein plugs in Santorini's duct. J Gastroenterol 1998;33:289-294.

12 Balakrishnan V, Chatni S, Radhakrishnan L, Narayanan VA, Nair P: Groove pancreatitis: a case report and review of literature. JOP 2007;8:592-597.

13 Yamaguchi K, Tanaka M: Groove pancreatitis masquerading as pancreatic carcinoma. Am J Surg 1992;163:312-318.

-14 Shudo R, Yazaki Y, Sakurai S, Uenishi H, Yamada H, Sugawara K, Okamura M, Yamaguchi K, Terayama H, Yamamoto Y: Groove pancreatitis: report of a case and review of the clinical and radiologic features of groove pancreatitis reported in Japan. Intern Med 2002;41:537-542.

-15 Mohl W, Hero-Gross R, Feifel G, Kramann B, Püschel W, Menges M, Zeitz M: Groove pancreatitis: an important differential diagnosis to malignant stenosis of the duodenum. Dig Dis Sci 2001;46:1034-1038.

-16 Itoh S, Yamakawa K, Shimamoto K, Endo T, Ishigaki T: CT findings in groove pancreatitis: correlation with histopathological findings. J Comput Assist Tomogr 1994;18:911915 .
17 Irie H, Honda H, Kuroiwa T, Hanada K, Yoshimitsu K, Tajima T, Jimi M, Yamaguchi K, Masuda K: MRI of groove pancreatitis. J Comput Assist Tomogr 1998;22:651-655.

18 Rahman SH, Verbeke CS, Gomez D, McMahon MJ, Menon KV: Pancreatico-duodenectomy for complicated groove pancreatitis. HPB (Oxford) 2007;9:229-234.

19 Blasbalg R, Baroni RH, Costa DN, Machado MC: MRI features of groove pancreatitis. AJR Am J Roentgenol 2007;189:73-80.

20 Levenick JM, Gordon SR, Sutton JE, Suriawinata A, Gardner TB: A comprehensive, casebased review of groove pancreatitis. Pancreas 2009;38:e169-e175.

21 Gabata T, Kadoya M, Terayama N, Sanada J, Kobayashi S, Matsui O: Groove pancreatic carcinomas: radiological and pathological findings. Eur Radiol 2003;13:1679-1684.

22 Casetti L, Bassi C, Salvia R, Butturini G, Graziani R, Falconi M, Frulloni L, Crippa S, Zamboni G, Pederzoli P: 'Paraduodenal' pancreatitis: results of surgery on 58 consecutives patients from a single institution. World J Surg 2009, E-pub ahead of print.

23 Isayama $\mathrm{H}$, Kawabe T, Komatsu Y, Sasahira N, Toda N, Tada M, Nakai Y, Yamamoto N, Hirano K, Tsujino T, Yoshida H, Omata M: Successful treatment for groove pancreatitis by endoscopic drainage via the minor papilla. Gastrointest Endosc 2005;61:175-178. 